\title{
Review: magnesium prophylaxis after cardiac surgery reduces the risk of arrhythmia and atrial fibrillation
}

Shiga T, Wajima Z, Inove T, et al. Magnesium prophylaxis for arrhythmias after cardiac surgery: a meta-analysis of randomized controlled trials. Am J Med 2004; 117:325-33.

Does magnesium prophylaxis after cardiac surgery reduce the risk of arrhythmia?

\section{METHODS}

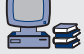

Data sources: Medline (1966 to June 2003), EMBASE/Excerpto Medica (1980 to June 2003), Cochrane Controlled Trials Register

(Issue 3, 2003), and hand searches of references of reports and reviews.

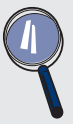

Study selection and assessment: randomised controlled trials (RCTs) in any language that compared a single, fixed dose of prophylactic magnesium (intravenous, central, or intracoronary administration) with control (placebo or routine care) after cardiac surgery. 2 independent reviewers assessed the methodological quality of individual trials using the 5 point Jadad scale.

Outcomes: atrial fibrillation (AF), supraventricular arrhythmia (SA) and ventricular arrhythmia (VA), serum magnesium concentration on postoperative day 1, length of hospital stay, myocardial infarction (MI), and mortality.

\section{MAIN RESULTS}

17 RCTs $(n=2069)$ met the selection criteria. 9 studies had Jadad scores of $4-5$ out of 5 , and 8 studies had scores $\leqslant 3$. Pooled serum magnesium concentration at 24 hours after surgery was higher in the magnesium group than in the control group (weighted mean difference [WMD] $1.1 \mathrm{mg} / \mathrm{dl}, 95 \%$ CI 0.7 to 1.4). Meta-analysis based on a random effects model showed that fewer patients who received magnesium had SAs, VAs, or AF compared with those who received the control intervention (table). The groups did not differ for length of hospital stay ( 7 trials, $\mathrm{n}=1227$, WMD $0.28 \mathrm{~d}, \mathrm{CI}-0.70$ to 1.27), perioperative MI (9 trials, $\mathrm{n}=1191$, relative risk [RR] 1.03, CI 0.52 to 2.05 ), or mortality ( 12 trials, $n=1588$, RR 0.97 , CI 0.43 to 2.20). Only 5 trials $(n=648)$ investigated side effects; no severe bradycardia or hypotension was reported.

\section{CONCLUSIONS}

Prophylactic treatment with magnesium after cardiac surgery is better than usual care or placebo for reducing the risk of atrial fibrillation and supraventricular and ventricular arrhythmias. Magnesium had no effect on length of hospital stay, myocardial infarction, or mortality.

For correspondence: Dr T Shiga, Department of Anesthesia, Chiba Hokuso Hospital, Nippon Medical School, Chiba, Japan. QZX02115@nifty.ne.jp; Shigat@aol.com

Source of funding: no external funding

\section{Commentary}

D patie search on the use of magnesium sulfate to suppress SAs and VAs in patients having cardiac surgery continues to provide conflicting results. The meta-analysis by Shiga et al establishes 2 points. Firstly, the findings of previous studies on the lack of effect of magnesium on patient mortality are supported; ${ }^{1}$ the effect of magnesium on hospital length of stay and overall patient outcomes requires further study in a large multicentre trial. ${ }^{2}$ In contrast to the findings of others, ${ }^{3}$ Shiga et al confirm the prophylactic antiarrhythmic properties of magnesium. Secondly, although magnesium may be less potent than other medications (ie, $\beta$-blockers, amiodarone, and sotalol) for suppressing arrhythmias in cardiac surgery patients, it may also be associated with fewer harmful side effects. However, only 5 studies monitored side effects. Limitations of the review are the small number of studies that met the selection criteria and variability among studies in magnesium administration (ie, frequency and concomitant administration of other medications).

The results of the meta-analysis by Shiga et al are relevant to nurses caring for cardiac surgery patients. The findings establish realistic outcomes and risk considerations for magnesium treatment. This information will be useful to nurses in their efforts to collaborate with physicians to match drug effectiveness with patient needs. Patients who have off-pump coronary artery surgery have fewer episodes of postoperative $\mathrm{AF}^{4}{ }^{5}$ compared with on-pump patients and therefore may require a less potent drug or no prophylactic antiarrhythmics.

As operative approaches evolve, additional research will be needed to clarify the use of magnesium. Advanced practice nurses who are involved at the bedside in collaborative care efforts are in an ideal position to lead research efforts that investigate medication timing, prescribing differences, side effects, and patient outcomes

\section{Gina Maiocco, RN, PhD, CCRN, CCNS} Wright State University Dayton, Ohio, USA

1 Magnesium in Coronaries (MAGIC) Trial Investigators. Early administration of intravenous magnesium to high-risk patients with acute myocardial infarction in the Magnesium in Coronaries (MAGIC) Trial: a randomised controlled trial. Lancet 2002;360:1189-96.

2 Roscoe A and Ahmed AB. A survey of peri-operative use of magnesium sulphate in adult cardiac surgery in the UK. Anaesthesia 2003;58:363-5.

3 Hazelrigg SR, Boley TM, Cetindag IB, et al. The efficacy of supplemental magnesium in reducing atrial fibrillation after coronary artery bypass grafting. Ann Thorac Surg 2004;77:824-30.

4 Raja S, Dreyfus G. Incidence of atrial fibrillation after off-pump and onpump coronary artery surgery: current best available evidence. Internet Journal of Thoracic and Cardiovascular Surgery 2003;6(2). http:// www.ispub.com/ostia/index.php?xmlFilePath= journals/ijtcvs/volon2/ afib.xml

5 Jayam VK, Flaker GC, Jones JW. Atrial fibrillation after coronary bypass: etiology and pharmacologic prevention. Cardiovasc Surg 2002;10:351-8.

Magnesium prophylaxis $v$ control (placebo or routine care) after cardiac surgery*

\begin{tabular}{|c|c|c|c|c|c|}
\hline \multirow[b]{2}{*}{ Outcomes at $1-30$ days } & \multirow[b]{2}{*}{ Number of trials ( $n$ ) } & \multicolumn{2}{|c|}{ Weighted event rates } & \multirow[b]{2}{*}{$\operatorname{RRR}(95 \% \mathrm{Cl})$} & \multirow[b]{2}{*}{ NNT (Cl) } \\
\hline & & Magnesium prophylaxis & Control & & \\
\hline Supraventricular arrhythmia & $16(2029)$ & $22 \%$ & $31 \%$ & $23 \%$ (7 to 37$)$ & 12 (8 to 22$)$ \\
\hline Ventricular arrhythmia & $10(1195)$ & $5 \%$ & $13 \%$ & $51 \%(13$ to 73$)$ & $13(7$ to 59$)$ \\
\hline Atrial fibrillation & $12(1649)$ & $19 \%$ & $28 \%$ & $29 \%$ (7 to 45$)$ & 12 (8 to 27$)$ \\
\hline
\end{tabular}

*Abbreviations defined in glossary; weighted event rates, RRR, NNT, and Cl calculated from data in article using a random effects model. 